Article

\title{
Neighborhood Approximations for Non-Linear Voter Models
}

\author{
Frank Schweitzer $^{1, *}$ and Laxmidhar Behera ${ }^{2}$ \\ ${ }^{1}$ Chair of Systems Design, ETH Zürich, Weinbergstrasse 58, 8092 Zürich, Switzerland \\ ${ }^{2}$ Department of Electrical Engineering, Indian Institute of Technology, 208016 Kanpur, India; \\ E-Mail: lbehera@iitk.ac.in \\ * Author to whom correspondence should be addressed; E-Mail: fschweitzer@ethz.ch; \\ Tel.: +41-44-632-83-50.
}

Academic Editor: J.A. Tenreiro Machado

Received: 2 September 2015 / Accepted: 4 November 2015 / Published: 10 November 2015

\begin{abstract}
Non-linear voter models assume that the opinion of an agent depends on the opinions of its neighbors in a non-linear manner. This allows for voting rules different from majority voting. While the linear voter model is known to reach consensus, non-linear voter models can result in the coexistence of opposite opinions. Our aim is to derive approximations to correctly predict the time dependent dynamics, or at least the asymptotic outcome, of such local interactions. Emphasis is on a probabilistic approach to decompose the opinion distribution in a second-order neighborhood into lower-order probability distributions. This is compared with an analytic pair approximation for the expected value of the global fraction of opinions and a mean-field approximation. Our reference case is averaged stochastic simulations of a one-dimensional cellular automaton. We find that the probabilistic second-order approach captures the dynamics of the reference case very well for different non-linearities, i.e., for both majority and minority voting rules, which only partly holds for the first-order pair approximation and not at all for the mean-field approximation. We further discuss the interesting phenomenon of a correlated coexistence, characterized by the formation of large domains of opinions that dominate for some time, but slowly change.
\end{abstract}

Keywords: opinion dynamics; voter model; pair approximation; higher-order probability distribution; cellular automata 


\section{Introduction}

The concept of entropy plays a key role in describing the transition between disorder and order. Specifically, the emergence of order in a random phase is indicated by a significant reduction of entropy. Calculating this reduction requires to know the probability of each possible system configuration that is compatible with the given system constraints-a challenging problem both methodologically and computationally. If we consider a system of $N$ elements each of which can be in one of two states-for example up and down spins in a physical system, or agents with opposite opinions in a social system, or agents with the two strategies cooperate or defect in an economic system-the number of possible configurations is $2^{N}$, which can be quite large. In fact, statistical physics was founded in the 19th century to provide an efficient solution based on the concept of statistical ensembles and state sums.

In this paper, we address the problem by proposing a stochastic approach that allows to decompose such probabilities for systems characterized by neighbor-neighbor interactions. Our candidate model to describe this interaction is the so-called voter model which is discussed in more detail in Section 3. In this model, agents are in one of two discrete states, $\sigma \in\{0,1\}$, denoted as "opinions". They change their opinion in response to the opinions in their neighborhood. In order to define such a neighborhood, we have chosen a one-dimensional cellular automaton (CA), in which consecutively numbered cells $i=1, \ldots, N$ represent agents (see Figure 1). We assume that the CA forms a ring to close the system. Each agent $i$ then has two neighbors $i-1, i+1$, i.e., their opinions $\theta_{i}$ form a triplet $T_{i}=\left\{\theta_{i-1}, \theta_{i}, \theta_{i+1}\right\}$. The second-order neighborhood that also takes the neighbors of $i-1, i+1$ into account, then results in a quintuplet of opinions $Q_{i}=\left\{\theta_{i-2}, \theta_{i-1}, \theta_{i}, \theta_{i+1}, \theta_{i+2}\right\}$.

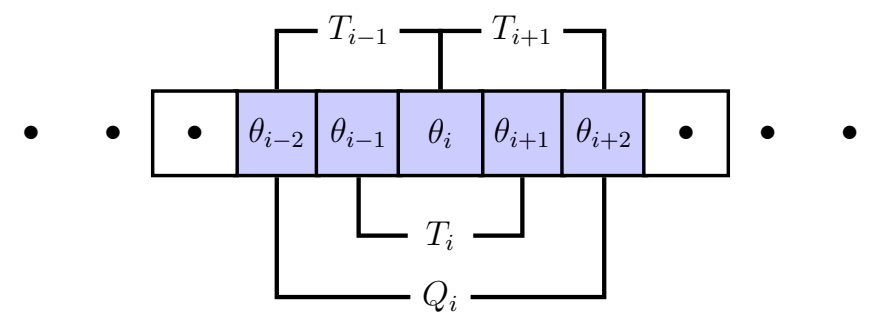

Figure 1. One-dimensional cellular automaton (CA) with triplet $\left(T_{i}\right)$ and quintuplet $\left(Q_{i}\right)$ definition of the neighborhood of agent $i$.

Our major assumption of the voter model is that changes in the opinion of agent $i$ are only caused by the first-order neighborhood. Specifically, the agent responds to the local frequency of opinions in the triplet that also includes its own opinion. However, because the dynamics of agent $i$ depends on its neighbors, it is also coupled to their dynamics, i.e., to the second-order neighborhood of agent $i$, and so forth. This specifically denotes the problem that we are going to discuss in this paper. In a stochastic approach, we are faced with a system of $N$ coupled dynamic equations for the probabilities $p\left(\theta_{i}, t\right)$ to find any agent $i$ with opinion $\theta_{i}$ at time $t$. This coupling exists only through the two neighbors of each agent, i.e., changes in a far distant cell only propagate slowly through the CA by means of neighbor-neighbor interactions. So, precisely, how large should be the neighborhood taken into account for the dynamics of the CA? Or, how far reaching are correlations in the changes of opinions? 
To answer this question, in this paper we propose three different analytic approximations for the dynamics which take different neighborhood sizes into account: zero-order-no correlations between neighboring agents, first-order-correlations between an agent and one of its nearest neighbors (so called pair approximation), second-order-correlations between an agent and its second-nearest neighbors. We test the validity of these dynamic approximations by comparing them to stochastic simulations of the CA, averaged over a number of runs.

Our emphasis is of course on the second-order approximation, which extends previous investigations. But we want to understand under which circumstances this approximation fares better than the simpler ones. Therefore, we have chosen different variants of the voter model, also known as nonlinear voter models. The non-linearity is with respect to the response to the local frequency of opinions. We further compare deterministic and stochastic dynamics, to show whether our analytic approximations can correctly predict the dynamics. Our variable of interest is the expected fraction of opinion 1, denoted as $\langle x(t)\rangle$, which is an aggregated variable for which we derive the dynamics based on a stochastic approach.

Our paper combines, and extends, previous investigations in different directions. One line of research refers to the approximation of higher-order probability distributions by means of lower-order distributions [1] which has been also applied to the voter model [2]. This implies a loss of (microscopic) information which can be quantified by means of information-theoretic measures [3]. The question whether the coarse-grained dynamics is still Markovian can be answered by analyzing emergent macroscopic memory effects using information-theoretic measures [4]. Such measures have also been applied to voter models [5]. In most cases, the Markov chain analysis becomes quite cumbersome and therefore is restricted to one-dimensional CA [6].

Another line of research considers different forms of neighborhood approximations specifically for binary state-dynamics (see [7] for a good overview), which also has been applied to the voter model, already [8]. We note that our pair approximation approach follows [8], but applies it here to a one-dimensional CA, which results in different expressions for $\langle x(t)\rangle$ and the correlations $c_{1 \mid 1}(t)$.

Compared to two-dimensional CA [9,10] or even complex networks [11,12], one could find one-dimensional CA too simple. But this judgment is in fact not justified. Already one-dimensional CA have proven to exhibit a really complex dynamics, with a chance to derive analytic expressions. Extensions of the simple voter model, for example the Sznajd model [13] or the $q$-voter model [14], could be thoroughly analyzed for one-dimensional CA.

The emphasis of our investigations is on the validity of the analytic approximations for nonlinear voter models. This non-linearity can be introduced in different ways. In References [15-17], the authors discuss it on the level of individual agents that respond to neighboring influences in a heterogeneous manner. Specifically, Stark et al. [16] assumes a heterogeneous inertia for agents to change their opinions, Castellano et al. [15] assumes a heterogeneous neighborhood size to influence agents, while Xiong et al. [17] assumes a heterogeneous weight for the influence of agents.

Compared to these approaches, we assume a homogeneous, but nonlinear response of agents on the local frequency of opinions. Specifically, we consider the majority rule, where the tendency of agents' to change their opinion increases with the frequency of the opposite opinion. The minority rule, on the other hand, assumes exactly the opposite, i.e., a decreasing tendency. This can be simply varied by one parameter $\alpha$, which however is assumed to be the same for all agents. 
Eventually, we would like to point out that we refrain from interpreting our model in a social context. Although agents are called "voters" and their states are called "opinions", the simplicity of the underlying assumptions does not justify to sell the model as a reflection of a social system. We see it rather as a very generic setup to better understand the impact of local feedback on emerging systemic properties, such as consensus (i.e., a "ferromagnetic" phase) or coexistence (i.e., a "paramagnetic" phase). But we acknowledge that, despite this basic limitations, the voter model has been applied in various context, e.g., to model investors' behavior in financial markets [18], emerging communication networks [19], or invasion of species [20]. A good overview of spin-type models in sociophysics is given in [21].

\section{Stochastic Approach}

\subsection{Defining the Cellular Automaton}

In this paper, we consider a one-dimensional CA consisting of $N$ cells, each of which is identified by the index $i \in N$ (see Figure 1). Each cell shall be characterized by a discrete value $\theta_{i}=\{0,1\}$, hence the total distribution of states is given by the vector $\Theta=\left\{\theta_{1}, \ldots, \theta_{i-2}, \theta_{i-1}, \theta_{i}, \theta_{i+1}, \theta_{i+2}, \ldots, \theta_{N}\right\}$. Assuming a torus space, each cell $i$ has a clearly defined neigborhood of first and second nearest neighbors, $\underline{\theta}_{i}^{\prime}=$ $\left\{\theta_{i-1}, \theta_{i+1}\right\}$ and $\underline{\theta}_{i}^{\prime \prime}=\left\{\theta_{i-2}, \theta_{i+2}\right\}$. The probability to find cell $i$ in state $\theta_{i}$ at time $t$ (where time shall be measured in discrete steps) is $p\left(\theta_{i}, t\right)$. Consequently, the conditional probabilities $p\left(\theta_{i} \mid \underline{\theta}_{i}^{\prime}, t\right)$ and $p\left(\theta_{i} \mid \underline{\theta}_{i}^{\prime}, \underline{\theta}_{i}^{\prime \prime}, t\right)$ describe the probability to find cell $i$ in state $\theta_{i}$ given that it has the first and second nearest neighbors $\underline{\theta}_{i}^{\prime}, \underline{\theta}_{i}^{\prime \prime}$.

Under Markov assumptions the Chapman-Kolmogorov equation holds for the probability $p\left(\theta_{i}, t\right)$ to find cell $i$ in state $\theta_{i}$ at time $t+1$ :

$$
p\left(\theta_{i}, t+1\right)=\sum_{\hat{\theta}_{i} \in \theta^{\star}} p\left[\left(\theta_{i}, t+1\right) \leftarrow\left(\hat{\theta}_{i}, t\right)\right] p\left(\hat{\theta}_{i}, t\right) .
$$

The propagator $p\left[\left(\theta_{i}, t+1\right) \leftarrow\left(\hat{\theta}_{i}, t\right)\right]$ denotes the transition probability to go from any given state $\hat{\theta}_{i}$ at time $t$ to the assumed state $\theta_{i}$ in the next time step, $t+1$. Here the summation is over all possible realizations $\theta^{\star}$ of $\hat{\theta}_{i}$, i.e., the $2^{1}$ possible states $\{0,1\}$. At this point, we make our 1 st assumption, namely that any change of $\theta_{i}$ depends on the nearest neighbors, given by $\underline{\theta}_{i}^{\prime}=\left\{\theta_{i-1}, \theta_{i+1}\right\}$. That means a triplet $T_{i}=\left\{\theta_{i-1}, \theta_{i}, \theta_{i+1}\right\}$ decides about the value of $\theta_{i}$ in the next time step. If we define the probability of a triplet configuration as

$$
p\left(T_{i}, t\right)=p\left(\theta_{i}, \underline{\theta}_{i}^{\prime}, t\right)=p\left(\theta_{i-1}, \theta_{i}, \theta_{i+1}, t\right),
$$

then the probability $p\left(\theta_{i}, t\right)$ results as the marginal distribution of the triplet probability:

$$
p\left(\theta_{i}, t\right)=\sum_{\underline{\theta}_{i}^{\prime} \in \underline{\underline{\prime}}^{\prime \star}} p\left(\theta_{i}, \underline{\theta}_{i}^{\prime}, t\right)=\sum_{T_{i} \in T^{\star}} p\left(\theta_{i} \mid T_{i}, t\right) .
$$

Here the summation is over all possible realizations $\underline{\theta}^{\prime \star}$ of the nearest neighborhood $\underline{\theta}_{i}^{\prime}$, i.e., $2^{2}$ different possibilities. $p\left(\theta_{i} \mid T_{i}, t\right)$ denotes the conditional probability to find $\theta_{i}$ as the focal cell given a triplet $T_{i}$, and the summation is over all possible realizations $T^{\star}$ of $T_{i}$, i.e., $2^{3}$ different possibilities. 
Based on the assumption that the nearest neighborhood matters, we can rewrite Equation (1) as

$$
p\left(\theta_{i}, t+1\right)=\sum_{\hat{\theta}_{i} \in \theta^{\star}} \sum_{\underline{\theta}_{i}^{\prime} \in \underline{\theta}^{\prime \star}} p\left[\left(\theta_{i}, t+1\right) \leftarrow\left(\hat{\theta}_{i} \mid \underline{\theta}_{i}^{\prime}, t\right)\right] p\left(\hat{\theta}_{i} \mid \underline{\theta}_{i}^{\prime}, t\right)
$$

where $p\left(\theta_{i} \mid \underline{\theta}_{i}^{\prime}, t\right)$ denotes the conditional probability to find the focal cell in state $\theta_{i}$ given the neighborhood $\underline{\theta}_{i}^{\prime}$. Using Bayes' rule, we can express this probability as:

$$
p\left(\theta_{i} \mid \underline{\theta}_{i}^{\prime}, t\right)=\frac{p\left(\theta_{i}, \underline{\theta}_{i}^{\prime}, t\right)}{p\left(\underline{\theta}_{i}^{\prime}, t\right)}=\frac{p\left(T_{i}, t\right)}{p\left(\underline{\theta}_{i}^{\prime}, t\right)} ; \quad \sum_{\underline{\theta}_{i}^{\prime} \in \underline{\theta}^{\prime \star}} p\left(\underline{\theta}_{i}^{\prime}, t\right)=1 .
$$

With this, we can eventually rewrite the Chapman-Kolmogorov Equation (1) for the single cell $i$ in terms of the triplet probabilities, $p\left(T_{i}, t\right)$ :

$$
p\left(\theta_{i}, t+1\right)=\sum_{T_{i} \in T^{\star}} p\left[\left(\theta_{i}, t+1\right) \leftarrow\left(T_{i}, t\right)\right] p\left(T_{i}, t\right) .
$$

The propagator $p\left[\left(\theta_{i}, t+1\right) \leftarrow\left(T_{i}, t\right)\right]$ describes the transition probabilities to go from any possible triplet $T_{i}$ to a state $\theta_{i}$ during the next time step.

This equation leaves us with the further specification of the triplet probability, $p\left(T_{i}, t\right)$. While, according to our 1 st assumption, the occurence of $\theta_{i}$ is just determined by the nearest neighbors $\underline{\theta}_{i}^{\prime}=\left\{\theta_{i-1}, \theta_{i+1}\right\}$, the occurrence of either $\theta_{i-1}$ or $\theta_{i+1}$ also depends on their nearest neighbors, i.e., the second nearest neighbors of $i, \underline{\theta}_{i}^{\prime \prime}=\left\{\theta_{i-2}, \theta_{i+2}\right\}$. That means a quintuplet $Q_{i}=\left\{\theta_{i-2}, \theta_{i-1}, \theta_{i}, \theta_{i+1}, \theta_{i+2}\right\}$ decides about the value of the triplet $T_{i}=\left\{\theta_{i-1}, \theta_{i}, \theta_{i+1}\right\}$ in the next time step. If we consider a quintuplet configuration $Q_{i}$, then we can define the triplet probability as the marginal probability:

$$
\begin{aligned}
p\left(Q_{i}, t\right) & =p\left(\theta_{i}, \underline{\theta}_{i}^{\prime}, \underline{\theta}_{i}^{\prime \prime}, t\right)=p\left(\theta_{i-2}, \theta_{i-1}, \theta_{i}, \theta_{i+1}, \theta_{i+2}, t\right), \\
p\left(T_{i}, t\right) & =\sum_{\underline{\theta}^{\prime \prime} \in \underline{\theta}^{\prime \prime \star}} p\left(\theta_{i}, \underline{\theta}_{i}^{\prime}, \underline{\theta}_{i}^{\prime \prime}, t\right)=\sum_{Q_{i} \in Q^{\star}} p\left(T_{i} \mid Q_{i}, t\right) .
\end{aligned}
$$

Here the summation is over all possible realizations $\underline{\theta}^{\prime \prime \star}$ of the nearest neighborhood $\underline{\theta}_{i}^{\prime \prime}$, i.e., $2^{2}$ different possibilities. $p\left(T_{i} \mid Q_{i}, t\right)$ denotes the conditional probability to find the focal triplet $T_{i}$ given a quintuplet $Q_{i}$, and the summation is over all possible realizations $Q^{\star}$ of $Q_{i}$, i.e., $2^{5}$ diffferent possibilities.

For the dynamics for the probability to find a triplet in state $T_{i}$ at time $t+1$, we can write a Chapman-Kolmogorov equation quite similar to Equation (6)

$$
p\left(T_{i}, t+1\right)=\sum_{Q_{i} \in Q^{\star}} p\left[\left(T_{i}, t+1\right) \leftarrow\left(Q_{i}, t\right)\right] p\left(Q_{i}, t\right) .
$$

The propagator $p\left[\left(T_{i}, t+1\right) \leftarrow\left(Q_{i}, t\right)\right]$ describes the transition probabilities to go from any possible quintuplet $Q_{i}$ to a triplet $T_{i}$ during the next time step.

In order to specify the quintuplet probability $p\left(Q_{i}, t\right)$, we may consider a neighborhood of seven, etc. However, following the above procedure repeatedly is neither convenient nor practicable since at the end we have to consider $2^{N}$ possible configurations. Therefore, in the next section, we present a more convenient method to determine the quintuplet probability distribution. 


\subsection{Quintuplet Approximation}

Instead of defining the quintuplet distribution in terms of higher-order probability distributions, following the procedure of the previous section, we now make our 2nd assumption by expressing these probabilities in terms of lower-order distributions, i.e., triplet distributions, this way arriving at a closed form description of the problem.

In the following we refer to the theory of approximating discrete probability distributions already developed in the late 1950 [1], which was also applied to voter model (VM) [2]. The idea is to approximate an $n$-th order probability distribution, $p\left(x_{1}, \ldots, x_{n}\right)$ by products containing the probabilities of given subsets, e.g., $p\left(x_{i}, x_{j}, x_{k}\right)$. It is known that such a product approximation contains at most $n$ terms, since every new term has to contain at least one variable $x_{i}$ not contained in previous terms. For example, the factorization $p\left(x_{1}, \ldots, x_{n}\right)=\prod_{i} p\left(x_{i}\right)$ already satisfies this condition, although it may be not a good approximation, since it holds only for ideal systems.

We note that the approximation procedure is not unique, i.e., there are various ways of constructing a product approximation. For example, taking $p\left(x_{1}, x_{2}, x_{3}\right)$, this can be approximated by $p\left(x_{1}, x_{3}\right) p\left(x_{2}\right)$ as well as by $p\left(x_{1}, x_{3}\right) p\left(x_{2} \mid x_{1}\right)$ or by $p\left(x_{1}, x_{3}\right) p\left(x_{2} \mid x_{3}\right)$. Which one of the above approximations is the most suitable has to be determined based on the information available about the triplet distribution $p\left(x_{1}, x_{2}, x_{3}\right)$. In some cases, there are measurements of this distribution, or additional information about the dependency between the $x_{i}$ as we will use below.

With reference to our quintuplet approximation using the triplets defined, we propose the following procedure: we first note that there are different triplets contained in the quintuplet configuration, for example: $T_{i}=\left\{\theta_{i-1}, \theta_{i}, \theta_{i+1}\right\}, T_{i-1}=\left\{\theta_{i-2}, \theta_{i-1}, \theta_{i}\right\}, T_{i+1}=\left\{\theta_{i}, \theta_{i+1}, \theta_{i+2}\right\}$. These are valid triplets because they consider the correct neighborhood relations, whereas for example $\left\{\theta_{i-1}, \theta_{i+1}, \theta_{i+2}\right\}$ would be inappropriate. Out of the valid triplets, we may choose for example the first one, $\left\{\theta_{i-1}, \theta_{i}, \theta_{i+1}\right\}$ and complete the product approximation as follows:

$$
p\left(\theta_{i-2}, \theta_{i-1}, \theta_{i}, \theta_{i+1}, \theta_{i+2}, t\right)=p\left(\theta_{i-1}, \theta_{i}, \theta_{i+1}, t\right) p\left(\theta_{i-2} \mid \theta_{i-1}, \theta_{i}, t\right) p\left(\theta_{i+2} \mid \theta_{i}, \theta_{i+1}, t\right) .
$$

In order to express the conditional probabilities using the triplet distribution, we apply Bayes' rule:

$$
p\left(\theta_{i-2} \mid \theta_{i-1}, \theta_{i}, t\right)=\frac{p\left(\theta_{i-2}, \theta_{i-1}, \theta_{i}, t\right)}{p\left(\theta_{i-1}, \theta_{i}, t\right)}
$$

which results in the final quintuplet approximation:

$$
p\left(\theta_{i-2}, \theta_{i-1}, \theta_{i}, \theta_{i+1}, \theta_{i+2}, t\right)=p\left(\theta_{i-1}, \theta_{i}, \theta_{i+1}, t\right) \frac{p\left(\theta_{i-2}, \theta_{i-1}, \theta_{i}, t\right)}{p\left(\theta_{i-1}, \theta_{i}, t\right)} \frac{p\left(\theta_{i}, \theta_{i+1}, \theta_{i+2}, t\right)}{p\left(\theta_{i}, \theta_{i+1}, t\right)} .
$$

We note that this product approximation is the only possible one, given that we have an ordered set of variables $\theta_{i}$ according to their neighborhood relations.

\subsection{Closed Form Dynamics}

Using the approximation explained above, we have reduced the higher-order description to the level of triplet distributions, $p\left(\theta_{i-1}, \theta_{i}, \theta_{i+1}, t\right)$, etc., which results in a closed form dynamics. We now have to 
specify the initial conditions for the distributions. While we are able to make any suitable assumption about the initial triplet distribution, we assume here that the occupation of the different cells is initially statistically independent, i.e.,

$$
p\left(\theta_{i-1}, \theta_{i}, \theta_{i+1}, 0\right)=p\left(\theta_{i-1}, 0\right) p\left(\theta_{i}, 0\right) p\left(\theta_{i+1}, 0\right)=p^{3}(\theta, 0) .
$$

For further investigations, we have set $p(\theta, 0)=p(1-\theta, 0)=1 / 2$.

In order to solve the above set of equations, we have two different possibilities: we can solve the dynamics (a) on the level of the distribution $p\left(\theta_{i}, t\right)$, Equation (6), or (b) on the level of the triplet distribution $p\left(T_{i}, t\right)$, Equation (9). Variant (a) requires more computational effort, since we have to replace any $p\left(T_{i}, t\right)$ as a marginal distribution of triplets, whereas in variant (b), given the reduction of the quintuplet distributions, we already have a closed form dynamics for the triplet distributions, which is computationally more efficient.

Variant (b), however, requires to know the transition probabilities on the level of the triplets, whereas these are usually specified on the level of single cell changes (see also the following section). Hence, we have to determine the propagators $p\left[\left(T_{i}, t+1\right) \leftarrow\left(Q_{i}, t\right)\right]$ in terms of $p\left[\left(\theta_{i}, t+1\right) \leftarrow\left(T_{i}, t\right)\right]$. Since our 1 st assumption specifies that the change of every cell in the triplet only depends on its nearest neighbors, we are able to factorize these transition probabilities:

$$
\begin{gathered}
p\left[\left(T_{i}, t+1\right) \leftarrow\left(Q_{i}, t\right)\right]=p\left[\left(\theta_{i-1}, t+1\right) \leftarrow\left(T_{i-1}, t\right)\right] \times \\
\quad \times p\left[\left(\theta_{i}, t+1\right) \leftarrow\left(T_{i}, t\right)\right] p\left[\left(\theta_{i+1}, t+1\right) \leftarrow\left(T_{i+1}, t\right)\right]
\end{gathered}
$$

This completes our closed form description. To calculate the time-dependent probability distribution of triplets, we have to insert Equations (12), (14) into Equation (9) and from the result calculate $p\left(\theta_{i}, t\right)$ as the marginal distribution, Equation (3). The only remaining task is now to specify the transition probabilities for single cells, $p\left[\left(\theta_{i}, t+1\right) \leftarrow\left(T_{i}, t\right)\right]$ at which point the VM comes into play.

\section{Nonlinear Voter Dynamics}

\subsection{Transition Rates}

The voter model, in its basic form, is applied to a population of $N$ agents, where each agent is characterized by a discrete value, its "opinion", $\theta_{i} \in\{0,1\}$. The opinion dynamics at the level of the agents works as follows: two agents $i$ and $j$ are randomly chosen from the population, and agent $j$ adopts the opinion of agent $i$, i.e., $\theta_{j}(t+1)=\theta_{i}(t)$. After $N$ such update events, time is increased by 1 (random sequential update).

The rather simplified rule limits the applications of the voter model to any real voting process or opinion dynamics. In a well mixed population, the probability for agent $j$ to adopt a given opinion $\sigma \in\{0,1\}$ is simply proportional to the global frequency $x_{\sigma}(t)$ of agents with the respective opinion:

$$
x_{\sigma}(t)=\frac{N_{\sigma}(t)}{N} ; \quad x(t) \equiv x_{1}(t)=1-x_{0}(t) ; \quad N=\sum_{\sigma} N_{\sigma}(t)=N_{0}(t)+N_{1}(t)=\text { const. }
$$

This dynamics is known to always converge to complete consensus, $x \rightarrow 1$ or $x \rightarrow 0$. The only interesting question is then how long it will take to reach this state dependent on the system size $N$ and the initial condition $x(0)$. 
This picture becomes more complex if instead of a well-mixed population a defined neighborhood for each agent is assumed. This can be a social network where agents have links to other agents, or simply a lattice where the neighborhood is given by the geometry that defines the nearest and second nearest neighbors of an agent. Then, instead of the global frequency, the probability of an agent to adopt a given opinion depends on the local frequency, $f_{i}^{\sigma}$, of this opinion in the neighborhood of that agent:

$$
f_{i}^{\sigma}=\frac{1}{3}\left[\delta_{\sigma, \theta_{i-1}}+\delta_{\sigma, \theta_{i}}+\delta_{\sigma, \theta_{i+1}}\right] ; \quad f_{i}^{(1-\sigma)}=1-f_{i}^{\sigma}
$$

Here $\delta_{x, y}$ means the Kronecker delta, which is 1 only for $x=y$ and zero otherwise. In this paper, we restrict ourselves to the one-dimensional regular lattice, where each agent is represented by a cell $i$ that has precisely two neighbors. Different from the basic voter rule, in the calculation of the local frequency we have taken the opinion $\theta_{i}$ of the focal cell $i$ into account. This adds some inertia to the dynamics as agent $i$ counts towards the local minority/majority. It also avoids stalemate situations where the two neighbors have different opinions.

The linear voter model would assume that the transition rate

$$
w\left(1-\theta_{i} \mid \theta_{i}=\sigma, f_{i}^{\sigma}\right)=f_{i}^{(1-\sigma)}
$$

of a focal cell $i$ to change its opinion from $\theta_{i}=\sigma$ to $1-\theta_{i}=1-\sigma$ is directly proportional to the local frequency $f_{i}^{(1-\sigma)}$, i.e., the opposite opinion in the neighborhood. The expression of Equation (17) is a specification of the more general transition probability $p\left[\left(\theta_{i}, t+1\right) \leftarrow\left(T_{i}, t\right)\right]$ used in Equation (14). It takes into account that for frequency dependent processes the change of $\theta_{i}$ depends not directly on the local distribution $T_{i}$, but only on the local frequency $f_{i}^{(1-\sigma)}$.

We are more interested in the non-linear case, where the local frequency still plays a role, however the response to the opposite opinion can be different. The linear voter model can then be generalized into a majority rule where the transition toward the opposite opinion monotonously increases with the respective local frequency. Different from this, the minority rule would assume that the transition toward the opposite opinion monotonously decreases with the respective local frequency, i.e., the minority opinion is favored. Eventually, we could also have mixed rules with a non-monotonous frequency dependence, which become possible only if the local frequency depends at least on the opinion of three agents, as it is the case in our model.

Instead of an analytical expression, we specify the transition rate $w\left(1-\theta_{i} \mid \theta_{i}=\sigma, f_{i}^{\sigma}\right)$ by using free parameters $\alpha_{0}, \alpha_{1}, \alpha_{2}, \alpha_{3}$ which cover all of the above cases in a general manner. With these, the transition rates shall be defined as follows:

\begin{tabular}{ccc}
\hline$f_{i}^{\sigma}$ & $f_{i}^{(1-\sigma)}$ & $w\left(1-\theta_{i} \mid \theta_{i}=\sigma, f_{i}^{\sigma}\right)$ \\
\hline 1 & 0 & $\alpha_{0}$ \\
$2 / 3$ & $1 / 3$ & $\alpha_{1}$ \\
$1 / 3$ & $2 / 3$ & $\alpha_{2}=1-\alpha_{1}$ \\
0 & 1 & $\alpha_{3}=1-\alpha_{0}$ \\
\hline
\end{tabular}

The general case of four independent transition rates in Equation (18) can be reduced to two transition rates $\alpha_{0}, \alpha_{1}$ by assuming a symmetry between the two states 0 and 1, i.e., $\alpha_{1}+\alpha_{2}=1, \alpha_{0}+\alpha_{3}=1$. The condition

$$
\alpha_{0} \leq \alpha_{1} \leq\left(1-\alpha_{1}\right) \leq\left(1-\alpha_{0}\right) ; \quad \alpha_{1} \leq 1 / 2
$$


then denotes the above mentioned majority rule, because the transition rate increases with an increasing fraction of the opposite opinion $(1-\sigma)$ in the neighborhood. This process is also known as positive freqency dependent invasion. In ecology, it means that individuals of abundant species have a better chance to survive.

Opposite to that, for the so-called negative freqency dependent invasion process the probability that cell $i$ shall switch to the opposite opinion $(1-\sigma)$ decreases with the fraction number of individuals of subpopulation $\sigma$ in the neighborhood. This implies

$$
\left(1-\alpha_{0}\right)>\left(1-\alpha_{1}\right)>\alpha_{1}>\alpha_{0} ; \quad \alpha_{1}>1 / 2
$$

In an ecological context negative frequency dependent invasion means that individuals of a rare subpopulation have a better chance to survive.

The rate $\alpha_{0}$ in Equation (18) applies for the case where cell $i$ is only surrounded by opinions of the same kind. In a deterministic model, there would be no force to change the current state. In a stochastic CA however all possible processes should have a certain non-zero probability to occur, therefore a rather small value $\alpha_{0} \equiv \varepsilon=10^{-4}$ is used to avoid absorbing states in the dynamics. We will then only vary the remaining rate $\alpha \equiv \alpha_{1}$, which is the only free parameter in the model. Table summarizes the different transition rates given the possible local configurations $T_{i}$. It should be read as follows: given that the local configuration is e.g., $T_{i}(t)=\{001\}$ at time $t$, the probability to find $T_{i}(t+1)=\{011\}$ is $\alpha$.

If $\alpha \leq 1 / 2$ this transition rate increases with $f_{i}^{(1)}$, hence it denotes a majority voting rule, otherwise it denotes a minority voting rule.

Now that the transition rates for the nonlinear voter model are specified, we have different ways to proceed: (1) We can run stochastic computer simulations of the one-dimensional CA, to get some intuition about the dynamics and the role of the parameter $\alpha$. This will be described in the following Section 3.2; (2) We can also derive an approximate macroscopic dynamics for the global frequency $x(t)$, which will be done in Sections 3.3 and 3.4 using two different approximations; (3) Eventually, we can use the closed-form description already derived in Section 2.3, to numerically calculate $x(t)$. The stochastic simulations will denote our reference case, used to compare the two different approximate macroscopic dynamics and the numerical calculations.

\subsection{Computer Simulations of the CA Model}

For a first insight into the dynamics, we have conducted stochastic simulations of the CA described above. In this section, we will only refer to particular runs, to show some snapshots of the dynamics, while in Section 4 also averaged simulation results of the global variables are discussed.

For our simulations, we have used a one-dimensional CA of $N=640$ cells with periodic boundary conditions, where all cells are simultaneously updated. The discrete time scale is defined by generations. We have checked that the main results do not change if $N$ is increased to 6400. The initial configuration of the CA refers to a homogeneous distribution (within discrete limits) of both opinions, i.e., initially each cell is randomly assigned one of the possible states, $\{0,1\}$, with a probability that is equal to the initial global frequency $x(t=0)$. At each time step, the transition rates are calculated for each cell according to Equation (18) and the values are compared with a random number rnd drawn from the intervall $(0,1)$. If $r$ nd is less than the calculated transition rate, the respective transition process is 
carried out, otherwise the cell remains in its current state. Since each transition only depends on the current local configuration, memory effects are not considered here.

It follows from the above description that the case $\alpha_{0}=\varepsilon=0$ and $\alpha=0$ refers to a deterministic positive majority voting rule, simply because the state of cell $i$ never changes unless the two nearest neighbor cells have adopted the same opinion. However, then, it will always change, such that all three cells have the same opinion. Similarly, a deterministic minority voting rule is described by $\varepsilon=0$, and $\alpha=1$.

The results of the computer simulations are illustrated in Figure 2. We first note the remarkable difference in the outcome between majority and minority voting. In the latter, cells change their state frequently, however no pattern evolves because the cell always adopts an opinion different from the two neighbors, if these have the same opinion. Thus, we see a rather random and unstructured pattern, in which both opinions coexist with a long-term fraction $x=0.5$. This overall behavior is also not changed in case of a stochastic dynamics.
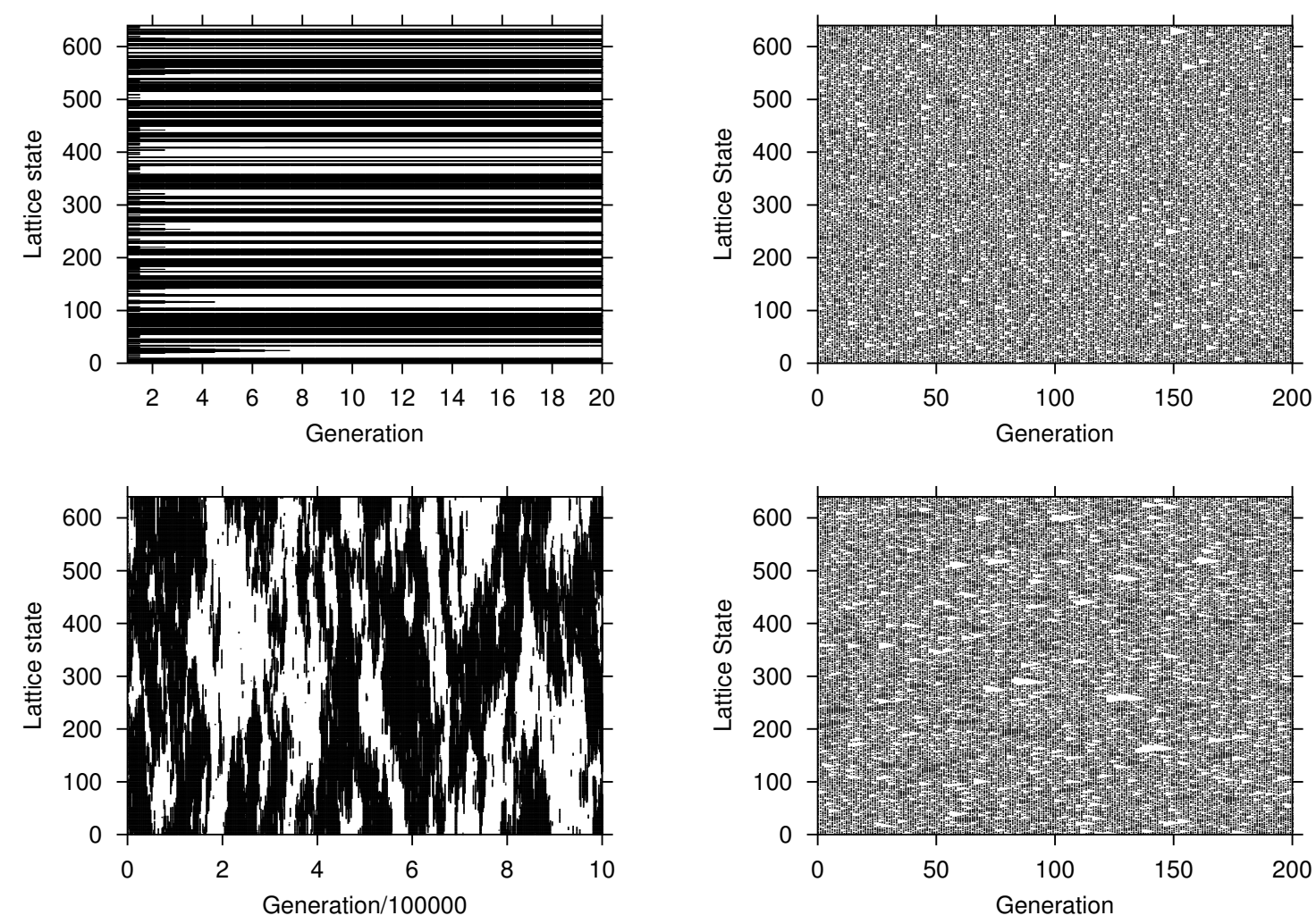

Figure 2. Evolution of the cellular automaton (CA) for different rules: (left column) majority voting; (right column) minority voting; (top row) deterministic dynamics; (bottom row) stochastic dynamics. Parameters: (top left) $\varepsilon=0.0, \alpha=0.0$, (top right) $\varepsilon=0.0, \alpha=1.0$, (bottom left) $\varepsilon=10^{-4}, \alpha=0.2$, (bottom right) $\varepsilon=10^{-4}, \alpha=0.8$. $N=640$, initial condition: $x(t=0)=0.5$, (black) indicates $\sigma=0$, (white) indicates $\sigma=1$.

In contrast, for the majority voting rule we observe the formation of local clusters of the same opinion. In the deterministic case, the resulting pattern becomes stationary very fast, such that the clusters remain rather small. In the stochastic case, however, we observe the interesting phenomenon that very large 
clusters of cells with the same opinion emerge. These domains do not grow up to system size because of the small perturbation $\varepsilon=10^{-4}$, that enables cells to adopt a different opinion even in a homogeneous neighborhood. The non-linearity ( $\alpha=0.2$ is different from the linear case $\alpha=1 / 3$ ) then amplifies such random deviations. This phenomenon has been discussed, for the case of a two-dimensional CA, in [8]. As a consequence, we observe a correlated coexistence of both opinions characterized by the facts that (i) each opinion at times is the majority and (ii) forms larger clusters, and (iii) the dynamics is always in non-equilibrium. This is clearly visible in the long-term behavior, shown in Figure 2 (bottom left).

In Section 4, we further investigate these different regimes numerically, after we have derived some appropriate approximations of the macroscopic dynamics.

\subsection{Derivation of the Macroscopic Dynamics}

A formal description for the dynamics of the CA starts with the probability $p\left(\theta_{i}, t\right)$ used in Equation (1). By means of

$$
\left\langle x_{\sigma}(t)\right\rangle=\frac{1}{N} \sum_{i=1}^{N} p\left(\theta_{i}=\sigma, t\right)
$$

we can obtain the key variable of the macroscopic dynamics $\left\langle x_{\sigma}(t)\right\rangle$, which is the expected global frequency of each opinion in the population. Note that, different from Equation (15) where $x_{\sigma}(t)$ is used, $\left\langle x_{\sigma}(t)\right\rangle$ describes the ensemble average over very many simulations. This is of some importance when interpreting the results. For the linear voter model, it is known that the system converges always to consensus, i.e., $x \rightarrow 0$ or $x \rightarrow 1$. If we use the initial condition $x(0)=0.5$, then in $50 \%$ of the cases we observe $x \rightarrow 0$, and in $50 \% x \rightarrow 1$. However, when averaging over all of these outcomes, we find $\langle x\rangle=0.5$.

Assuming a master equation for the dynamics of $p\left(\theta_{i}, t\right)$ with the transition rates specified in Equation (18), we can derive a rate equation for $\left\langle x_{\sigma}\right\rangle$ as discussed in detail in [8]:

$$
\frac{d}{d t}\left\langle x_{\sigma}(t)\right\rangle=\sum_{\underline{\sigma}^{\prime}}\left[w\left(\sigma \mid(1-\sigma), \underline{\sigma}^{\prime}\right)\left\langle x_{(1-\sigma), \underline{\sigma}^{\prime}}(t)\right\rangle-w\left(1-\sigma \mid \sigma, \underline{\sigma}^{\prime}\right)\left\langle x_{\sigma, \underline{\sigma}^{\prime}}(t)\right\rangle\right]
$$

Here, the summation is over all possible $2^{n}$ opinion patterns $\underline{\sigma}^{\prime}$ for the neighborhood of cell $i$. These are binary strings $\sigma_{i-1} \sigma_{i+1}$ that indicate the particular values of the nearest neighbors $i-1, i+1$. With $n=2$ neighbors, these would be $00,01,10,11$. Together with the possible state for cell $i, i . e ., \sigma=0$ or $\sigma=1$, the respective local frequencies $f_{i}^{\sigma}$ can be derived according to Equation (16), such that all transition rates in Equation (22) are specified. The solution of Equation (22) would however require the computation of the averaged global frequencies $\left\langle x_{1, \underline{\sigma}^{\prime}}\right\rangle$ and $\left\langle x_{0, \underline{\sigma}^{\prime}}\right\rangle$ for all possible opinion patterns $\underline{\sigma}^{\prime}$ over time, which would be a tremendous effort. Therefore, in [8] two analytic approximations have been discussed to solve this problem. Here, we only summarize the results.

In the first approximation, the so-called mean-field limit, the state of each cell does not depend on the opinion of its neighbors, but is only influenced via a mean field. In this case the opinion distribution factorizes:

$$
\left\langle x_{\sigma, \underline{\sigma}^{\prime}}\right\rangle=\left\langle x_{\sigma}\right\rangle \prod_{j=1}^{n}\left\langle x_{\sigma_{j}}\right\rangle
$$


and we find with $\langle x\rangle \equiv\left\langle x_{1}\right\rangle=1-\left\langle x_{0}\right\rangle$ for the macroscopic dynamics, Equation (22) in the mean-field limit:

$$
\frac{d}{d t}\langle x(t)\rangle=\sum_{\underline{\sigma}^{\prime}}\left[w\left(1 \mid 0, \underline{\sigma}^{\prime}\right)(1-\langle x\rangle) \prod_{j=1}^{n}\left\langle x_{\sigma_{j}}\right\rangle-w\left(0 \mid 1, \underline{\sigma}^{\prime}\right)\langle x\rangle \prod_{j=1}^{n}\left\langle x_{\sigma_{j}}\right\rangle\right]
$$

For the calculation of the $\left\langle x_{\sigma_{j}}\right\rangle$ we have to look at each possible opinion pattern $\underline{\sigma}^{\prime}$. The mean-field approach assumes that the occurence of each 1 or 0 in the pattern can be described by the global frequencies $x$ and $(1-x)$, respectively (for simplicity, the abbreviation $x \equiv\langle x\rangle$ will be used in the following). Taking the example 100, i.e., $\sigma_{i}=0$ for cell $i$ and $\sigma_{i-1}=1, \sigma_{i+1}=0$ for its neighbors, would result in $(1-\langle x\rangle)\left\langle x_{\sigma_{i-1}}\right\rangle\left\langle x_{\sigma_{i+1}}\right\rangle=x(1-x)^{2}$. Inserting further the transition rates, Equation (18), we find this way the equation for the mean-field dynamics as:

$$
\frac{d x}{d t}=\varepsilon\left[(1-x)^{3}-x^{3}\right]+(1-3 \alpha) x(1-x)(2 x-1)
$$

The fixed points of the mean-field dynamics can be calculated from Equation (25) by means of $\dot{x}=0$. In the limit $\varepsilon=0$, we find:

$$
x^{(1)}=0 ; \quad x^{(2)}=1 ; \quad x^{(3)}=0.5
$$

The three stationary solutions denote either consensus toward one of the opinions, or coexistence of both opinions with an equal share. In order to verify the stability of the solutions, we have further investigated the Jacobian $d / d x$ of Equation (25). The results can be concluded as follows: Below a critical reinforcement, $0<\alpha<1 / 3, x=0$ and $x=1$ are stable attractors and $x=0.5$ is the separatrix. Above the critical reinforcement, $1 / 3<\alpha<1, x=0.5$ becomes the stable attractor, while $x=0$ and $x=1$ are unstable. We will come back on this after discussing the second approximation.

\subsection{Pair Approximation}

The mean-field approximation assumes that the local occurrence of opinions is determined by the global frequencies rather than by local interactions. A better approximation should take local correlations between neighboring cells into account. Our second approximation, the so-called pair approximation, is based on the assumption that the state of each cell $i$ is only correlated to the states of each of its nearest neighbors, separately. i.e., the two neighbors are only correlated through the focal cell and not to each other. Therefore, the neighborhood $\sigma_{i-1} \sigma_{i} \sigma_{i+1}$ is decomposed in pairs of nearest neigbor cells, $\sigma_{i} \sigma_{i+1}$, $\sigma_{i-1} \sigma_{i}$ which are called doublets. The expected value of the global frequency of doublets is denoted as $\left\langle x_{\sigma, \sigma^{\prime}}\right\rangle$ where $\sigma$ refers to the focal cell and $\sigma^{\prime}$ to one of its neighbors.

We can then approximate the global frequency of a specific opinion pattern $\left\langle x_{\sigma, \underline{\sigma}^{\prime}}\right\rangle$ in Equation (22) as:

$$
\left\langle x_{\sigma, \underline{\sigma}^{\prime}}\right\rangle=\left\langle x_{\sigma}\right\rangle \prod_{j=1}^{n} c_{\sigma_{j} \mid \sigma}
$$

where the $c_{\sigma \mid \sigma^{\prime}}$ are the correlations:

$$
c_{\sigma \mid \sigma^{\prime}}:=\frac{\left\langle x_{\sigma, \sigma^{\prime}}\right\rangle}{\left\langle x_{\sigma^{\prime}}\right\rangle}
$$


that depend on the doublet frequency $\left\langle x_{\sigma, \sigma^{\prime}}\right\rangle$ and the global frequency of opinions $\left\langle x_{\sigma^{\prime}}\right\rangle$ neglecting higher order correlations. $c_{\sigma \mid \sigma^{\prime}}$ can be interpreted as the conditional probability that a randomly chosen nearest neighbor of a cell in state $\sigma^{\prime}$ is in state $\sigma$. With the relations:

$$
\left\langle x_{\sigma^{\prime}}\right\rangle c_{\sigma \mid \sigma^{\prime}}=\left\langle x_{\sigma}\right\rangle c_{\sigma^{\prime} \mid \sigma} ; \sum_{\sigma^{\prime} \in\{0,1\}} c_{\sigma^{\prime} \mid \sigma}=1
$$

and using again $\langle x\rangle \equiv\left\langle x_{1}\right\rangle$, these correlations can be expressed in terms of only $c_{1 \mid 1}$ and $\langle x\rangle$ as follows:

$$
c_{0 \mid 1}=1-c_{1 \mid 1} ; \quad c_{1 \mid 0}=\frac{\langle x\rangle\left(1-c_{1 \mid 1}\right)}{1-\langle x\rangle} ; \quad c_{0 \mid 0}=\frac{1-2\langle x\rangle+\langle x\rangle c_{1 \mid 1}}{1-\langle x\rangle}
$$

We then find for the macroscopic dynamics, Equation (22), in pair approximation:

$$
\frac{d}{d t}\langle x(t)\rangle=\sum_{\underline{\sigma}^{\prime}}\left[w\left(1 \mid 0, \underline{\sigma}^{\prime}\right)(1-\langle x\rangle) \prod_{j=1}^{n-1} c_{\sigma_{j} \mid \sigma}-w\left(0 \mid 1, \underline{\sigma}^{\prime}\right)\langle x\rangle \prod_{j=1}^{n-1} c_{\sigma_{j} \mid(1-\sigma)}\right]
$$

With respect to Equation (30), Equation (31) now depends on two variables, $\langle x\rangle$ and $c_{1 \mid 1}$. In order to derive a closed description, we need an additional equation for $\dot{c}_{1 \mid 1}$, that can be obtained from Equation (28):

$$
\frac{d c_{1 \mid 1}}{d t}=-\frac{c_{1 \mid 1}}{\langle x\rangle} \frac{d}{d t}\langle x\rangle+\frac{1}{\langle x\rangle} \frac{d}{d t}\left\langle x_{1,1}\right\rangle
$$

Equation (32) requires additionally the time derivative of the global doublet frequency $\left\langle x_{1,1}\right\rangle$. We note that the three coupled equations for $\langle x\rangle, c_{1 \mid 1}$ and $\left\langle x_{1 \mid 1}\right\rangle$ can be easily solved numerically. In the Appendix, we have derived explicit expressions for these equations for the one-dimensional CA discussed here, using the transition rates of Equation (18).

\section{Numerical Comparison of the Approximations}

\subsection{Computational Procedure}

In this section, we aim at a comparison of the different approaches developed so far. We recall that our reference case is the stochastic simulation of the CA, where some sample runs were already presented in Section 3.2. To allow a comparison with the other approaches that predict the expected behavior, we will average the CA simulations over 50 independent runs. The initial condition is given by the global fraction of opinion $1, x(t=0)$, which is realized as a uniform random distribution of the opinions as described already in Section 3.2. Thus, the initial value for the pair correlation is $c_{1 \mid 1}(t=0)=x(t=0)$. To decide when the CA simulations have reached stationarity, we verify that changes of $\langle x(t)\rangle$ are less than $1 / \sqrt{N}$.

The dynamics of the reference case (a) shall be compared to the two other dynamic approximations, (b) quintuplet approximation (see Section 2.3) and (c) pair approximation (see Section 3.4 and Appendix). To facilitate the understanding, we summarize here the computational procedure.

For the quintuplet approximation our starting point is Equation (9) which contains two terms that need a further approximation. The quintuplet probability $p\left(Q_{i}, t\right)$ can be decomposed in terms of triplet probabilities by means of Equation (12). The propagator $p\left[\left(T_{i}, t+1\right) \leftarrow\left(Q_{i}, t\right)\right]$ can be decomposed 
in terms of propagators $p\left[\left(\theta_{i}, t+1\right) \leftarrow\left(T_{i}, t\right)\right]$ by means of Equation (14). The latter are given as the transition rates of the VM in Table 1.

Table 1. Transition probabilities for $\theta_{i}$ given different local configurations. $\alpha \leq 1 / 2$ denotes majority voting rules, $\alpha>1 / 2$ denotes minority voting rules.

\begin{tabular}{ccccc}
\hline $\boldsymbol{\theta}_{\boldsymbol{i}}(\boldsymbol{t})=\mathbf{1}$ & $\boldsymbol{f}_{\boldsymbol{i}}^{(\mathbf{1})}$ & $\boldsymbol{f}_{\boldsymbol{i}}^{(\mathbf{0})}$ & $\boldsymbol{w}\left(\mathbf{0} \mid \mathbf{1}, \boldsymbol{f}_{\boldsymbol{i}}^{(\mathbf{1})}\right)$ & $\boldsymbol{t}+\mathbf{1}$ \\
\hline 010 & $1 / 3$ & $2 / 3$ & $1-\alpha$ & 000 \\
011 & $2 / 3$ & $1 / 3$ & $\alpha$ & 001 \\
110 & $2 / 3$ & $1 / 3$ & $\alpha$ & 100 \\
111 & 1 & 0 & $\varepsilon$ & 101 \\
\hline $\boldsymbol{\theta}_{\boldsymbol{i}}(\boldsymbol{t})=\mathbf{0}$ & $\boldsymbol{f}_{\boldsymbol{i}}^{(\mathbf{1})}$ & $\boldsymbol{f}_{\boldsymbol{i}}^{(\mathbf{0}}$ & $\boldsymbol{w}\left(\mathbf{1} \mid \mathbf{0}, \boldsymbol{f}_{i}^{(\mathbf{1})}\right)$ & $\boldsymbol{t}+\mathbf{1}$ \\
\hline 000 & 0 & 1 & $\varepsilon$ & 010 \\
001 & $1 / 3$ & $2 / 3$ & $\alpha$ & 011 \\
100 & $1 / 3$ & $2 / 3$ & $\alpha$ & 110 \\
101 & $2 / 3$ & $1 / 3$ & $1-\alpha$ & 111 \\
\hline
\end{tabular}

Let us illustrate the computation by one example. A quintuplet has five states and hence $2^{5}=32$ configurations denoted as $Q^{\star}$ in Equation (9). Each of these possible configurations will contribute to the triplet distribution according to Equation (9). Let us for the quintuplet take the sample configuration $\{01101\}$ and for the triplet $\{000\}$. The right-hand side of Equation (9) then reads:

$$
\begin{array}{r}
p[(0, t+1) \leftarrow(011, t)] p[(0, t+1) \leftarrow(110, t)] p[(0, t+1) \leftarrow(101, t)] \times \\
\times \frac{p(011, t) p(110, t) p(101, t)}{p(11, t) p(10, t)}
\end{array}
$$

In order to determine $p(000, t+1)$ according to Equation (9), we have to sum up over the 32 different quintuplet configurations. And we have to follow this procedure 8 times, because there are $2^{3}=8$ possible triplet configurations. At the end, we arrive at a new triplet distribution, which serves as the starting point for the next generation, and so forth.

Regarding the computational effort, it is comparably low because of the straight forward calculation. Because in the VM the transition rates do not depend on the lattice position $i$ but rather on the local frequencies $f_{i}$, it would be even possible to reduce the higher order approximation to a framework of frequencies of motives $\{000\},\{001\}$, etc. However, to test the resulting improvement in computation speed is not the aim of this paper.

For the pair approximation, we repeat again that we have to solve a system of three coupled equations for $\langle x\rangle, c_{1 \mid 1}$ and $\left\langle x_{1 \mid 1}\right\rangle$.The Appendix provides explicit expressions for the involved quantities, where the transition rates are obtained from Table 1. 


\subsection{Prediction of the Stationary Solution}

As a first test of the validity of our approximations, we focus on the correct prediction of the stationary solution, $\left\langle x^{\text {stat }}\right\rangle$. We have two parameters to vary: (i) the initial condition $x(t=0)$ which is changed from 0.1 to 0.9 in steps of 0.1 ; (ii) the parameter $\alpha$ that decides about majority and minority voting.

For the majority voting, the results are shown in Figure 3 for the deterministic $(\alpha=0)$ and the stochastic $(\alpha=0.2)$ case. There are two remarkable observations. First, there is a noticeable difference between the deterministic and the stochastic outcome. In the latter, for the reference case, we always observe $\left\langle x^{\text {stat }}\right\rangle=0.5$, regardless of the initial condition. This is understandable also from Figure 2 (lower left), where we observe the correlated coexistence of the two opinions. The small disturbance $\varepsilon=10^{-4}$ acts as a repulsion from consensus and averaging over the large opinion domains in the long-term limit leads to the balance between the two opinions. In the deterministic case, however, the dynamics freezes really fast, within the first 20 generations, which is also shown in Figure 2 (upper left). Hence, not many deviations from $x(t=0)$ can happen. However, $\left\langle x^{\text {stat }}\right\rangle$ is slightly below (for $x(t=0)<0.5$ ) or above (for $x(t=0)>0.5$ ) this value, which indicates the trend towards consensus.
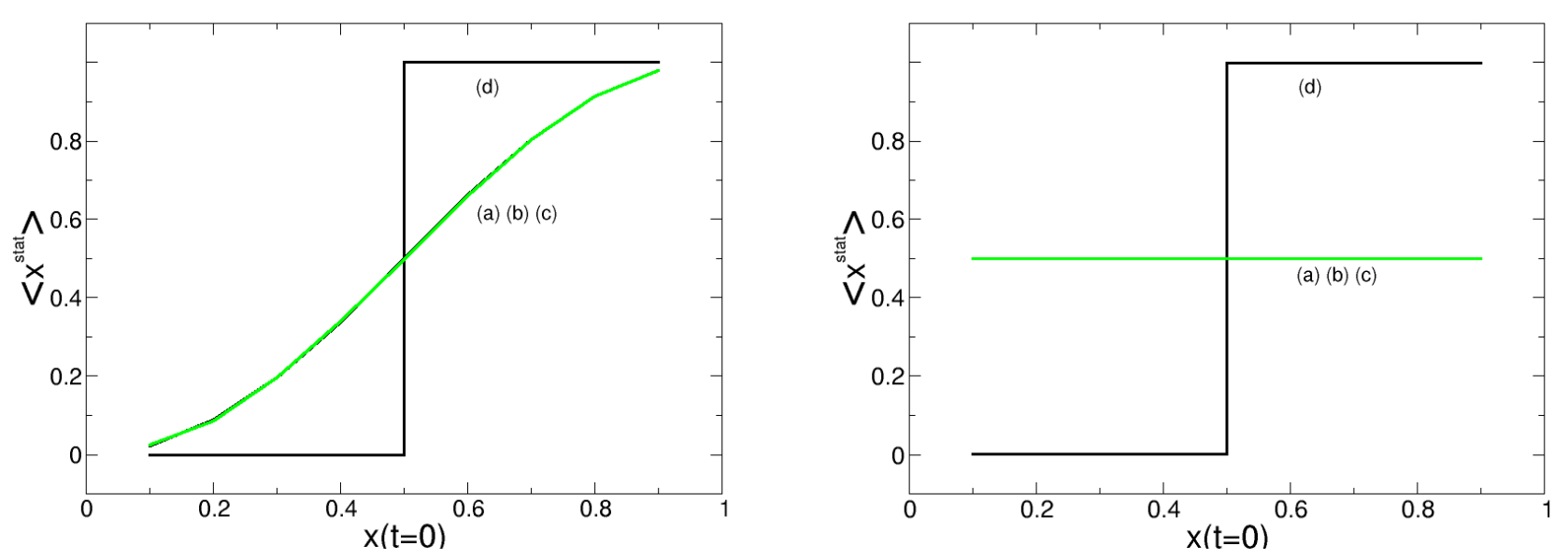

Figure 3. Stationary solution $\left\langle x^{\text {stat }}\right\rangle$ for majority voting. (left) deterministic case, $\alpha=0$, $\varepsilon=0$; (right) stochastic case, $\alpha=0.2, \varepsilon=10^{-4}$. Curves from different approaches:

(a) cellular automaton (CA) simulation (reference case, green); (b) quintuplet approximation;

(c) pair approximation; (d) mean-field approximation.

The second remarkable observation is the difference between the mean-field approximation (d) and the other approximations (b), (c) in predicting the stationary value. Regardless of the details of the dynamics, the mean-field approximation always predicts consensus for the opinion that was initially the majority, i.e., $x^{\text {stat }}=0$ for $x(t=0)<0.5$ and vice versa. The quintuplet and the pair approximation, however, correctly predict the expected stationary solution, as the match with the reference case ((a), green curve) indicates.

Hence, we can conclude that the mean-field approximation is not suitable to describe the dynamics of the one-dimensional CA for majority voting, which is not very surprising. But we are more interested to find out to what extend the pair and the quintuplet approximation, which both predict the expected 
stationary solution correctly for both the deterministic and the stochastic case, are also able to describe the dynamics toward the stationary state correctly. This is discussed in the following section.

We note that the minority voting rule, not further discussed here, always leads to symmetric coexistence of both opinions, $x^{\text {stat }}=0.5$, for each initial condition. This was already indicated in Figure 2 (right column). For minority voting, the mean-field approximation indeed predicts the correct stationary value, $\langle x\rangle=0.5$, but it miserably fails for majority voting.

\subsection{Prediction of the Time-Dependent Solution}

Again, we are interested in the dynamics of the expected fraction of opinion $1,\langle x(t)\rangle$. We concentrate only on the stochastic dynamics, since the deterministic dynamics freezes very fast. In Figure 4, we compare the validity of our two different approximations, the pair and the quintuplet approximation, with the reference case, the stochastic CA simulations, for both the majority and the minority voting rule.
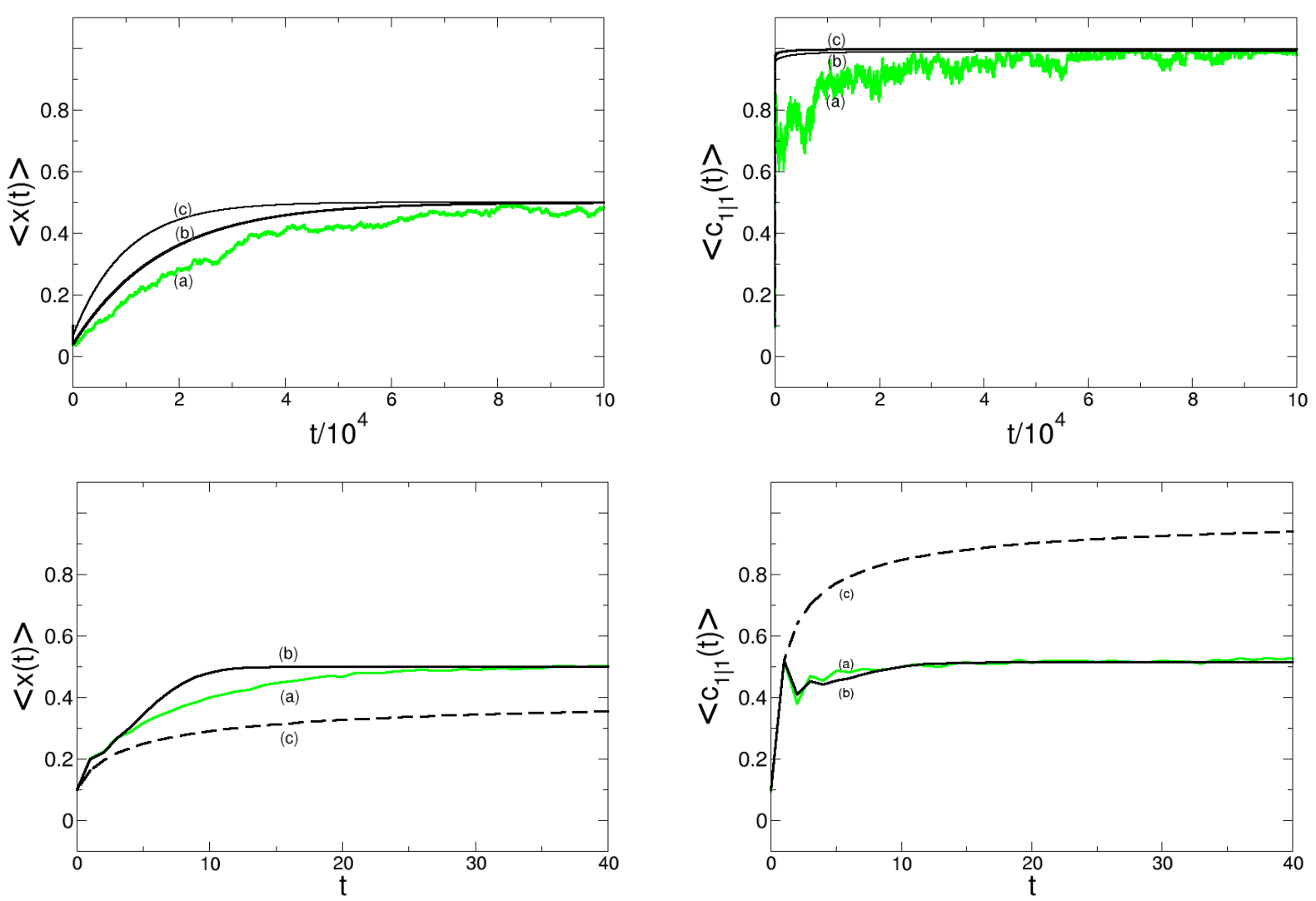

Figure 4. Dynamics of the expected frequency $\langle x(t)\rangle$ and the correlation $c_{1 \mid 1}(t)$. (upper row) majority voting, parameters: $\alpha=0.2, \varepsilon=10^{-4}, x(t=0)=0.1, c_{1 \mid 1}(t=0)=0.1$. (lower row) minority voting, parameters: $\alpha=0.8, \varepsilon=10^{-4}, x(t=0)=0.1, c_{1 \mid 1}(0)=0.1$. Curves from different approaches: (a) cellular automaton (CA) simulation (reference case, green); (b) quintuplet approximation; (c) pair approximation.

For the case of majority voting, all the three curves for $\langle x(t)\rangle$ actually start at $x=0.1$, but then decrease rapidly during the first few generations (which cannot be noticed on the long time scale), before 
they steadily evolve toward the quasistationary state $\langle x\rangle=0.5$. This kind of overshooting in the very initial phase can be also observed for other extreme initial conditions, e.g., $x=0.9$. In this case $\langle x(t)\rangle$ will first increase before settling at $\langle x\rangle=0.5$. We see that the two approximations reach this state much faster than the simulations. While this gap is noticable in particular in the beginning, the quintuplet approximation fares better than the pair approximation. The evolution of the correlation term $c_{1 \mid 1}(t)$ starts at $c_{1 \mid 1}=0.1$ and reaches values near one very quickly. This indicates the formation of large domains of the same opinion, i.e., domains with different opinions are well separated, but can coexist. However, we should notice that the stochastic simulations do not reach the value of 1 , because there is never a real consensus. Again, in the beginning, there is some discrepancy between the approximations and the simulations, but the quintuplet approximation is slightly more accurate.

For the case of minority voting, we notice that the quintuplet approximation fares considerably better than the pair approximation. The latter fails to predict the dynamics for both $\langle x(t)\rangle$ and $\left\langle c_{1 \mid 1}(t)\right\rangle$, whereas the quintuplet approximation is almost exact, except for a short time interval after the beginning, where it predicts a faster relaxation for $\langle x(t)\rangle$. Values of $\langle x\rangle=0.5$ and $\left\langle c_{1 \mid 1}\right\rangle=0.5$ clearly indicate a random pattern as also shown in the snapshots of Figure 2.

\section{Conclusions}

With our investigations of the one-dimensional voter model, we want to achieve two goals: (i) a better understanding of the non-linearity in the voter dynamics that was mostly studied as a linear model, only; (ii) a probabilistic description, and possible approximations, of the dynamics for the fraction of opinion $1, x(t)$.

The non-linearity can be easily expressed by means of a free parameter $\alpha$. The critical value $\alpha^{c}=1 / 3$ distinguishes between two different rules, majority voting $\left(\alpha>\alpha^{c}\right)$ and minority voting $\left(\alpha<\alpha^{c}\right)$, whereas $\alpha=\alpha^{c}$ refers to the border case of the linear voter model. It is known from well-mixed populations that majority voting should result in consensus, i.e., the asymptotic dominance of only one opinion, whereas minority voting should result in coexistence, i.e., the occurrence of both opinions in different fractions.

Our main focus was the role of local correlations in determining this outcome. For this we have used one-dimensional cellular automata (CA) in which each cell $i$, characterized by its opinion $\theta_{i}$, has a defined neighborhood of two cells with possibly different opinions, denoted as triplet. The transition probability of a cell to change its opinion is then determined by the local frequency of opinions in its neighborhood (including the focal cell) and the non-linear response to this information, expressed by means of $\alpha$. The values of $\alpha$ define a certain probability to switch to the opposite opinion, i.e., we can use them to switch between a deterministic $(\alpha=0,1)$ or a stochastic $(\alpha \neq 0,1)$ dynamics. In the latter case, we have further assumed a very small probability $\alpha_{0} \equiv \varepsilon=10^{-4}$ to perturb a state of complete consensus, which allows a non-stationary dynamics of the CA as shown in Figure 2 (bottom right).

While the minority rule only results in random coexistence of the two different opinions, the majority rule generates more interesting results. In particular, we observe a correlated coexistence characterized by the formation of large domains of the same opinion which change continuously. i.e., we have a 
non-equilibrium dynamics in which each opinion, for a certain time, can form large clusters of the majority opinion.

The question then is how to describe this dynamics mathematically. In this paper, we follow a probabilistic approach, i.e., each cell has a certain probability of a given opinion $\sigma \in\{0,1\}$, which also depends on the probabilities of the nearest neighbors, second-nearest neighbors, and so forth. In order to close the dynamics, we have proposed three different approximations at different levels of the description.

The first level is the aggregated description in terms of the global fraction of opinion 1, $x(t)$, for which we derive a dynamics for the expected value, $\langle x(t)\rangle$. On this level, we discuss two approximations. The simplest one is the mean-field approximation, in which no correlations between neighboring states are considered. So, we call this the zero-order approximation. It gives us a prediction for $\langle x(t)\rangle$ derived from the well-mixed case. In contrast, the pair approximation considers a correlation between a cell and its neighbor, i.e., the triplet consisting of a cell and its two neighbors is decomposed in two cell-neighbor pairs. Correlations between neighbors are not considered, so we call this the 1st-order approximation. Hence, we have a prediction for $\langle x(t)\rangle$ coupled to the dynamics of the pair correlations $c_{1 \mid 1}(t)$.

The third approximation does not refer to the aggregated level, but to the stochastic dynamics of a triplet, i.e., a cell with its two neighbors, that is determined by the larger neighborhood of a quintuplet, i.e., considers also the second nearest neighbors of the cell. Therefore, we call this the 2nd-order approximation. Using certain assumptions, we are able to provide a closed form dynamics for this larger neighborhood in terms of a probabilistic equation.

To compare the validity of these mathematical approximations, we use as a reference case stochastic computer simulations of the one-dimensional CA, which are averaged over a larger number of runs. We have discussed the majority and the minority voting, as well as the deterministic and the stochastic dynamics, separately.

In conclusion, we can summarize that the zero-order approximation only predicts the stationary outcome of the minority voting correctly, but fails for the majority voting rule, both with respect to the dynamics and the stationary outcome.

The first-order approximation performs comparably well in comparison to the second-order approximation only for the majority voting. The stationary outcome is correctly predicted both for the deterministic and the stochastic case, also the dynamics is covered fairly good. However, the first-order approximation fails to predict the dynamics of the minority voting. This case is only well covered by the second-order approximation that gives not only a correct description of $\langle x(t)\rangle$, but also of the pair correlations $\left\langle c_{1 \mid 1}(t)\right\rangle$.

Commenting specifically on the correlations, we recall again that both the first and second-order approximations lead to comparably good asymptotic results only for the majority voting. But they clearly predict a faster formation of domains, i.e., a convergence to their stationary value, as compared to the CA simulations. This limits their usability to fully understand the emergence of long-range correlations. In the case of minority voting, our first-order approximation fails, while the second-order approximation for $\left\langle c_{1 \mid 1}(t)\right\rangle$ could be even seen as accurate in its computational prediction. This is not so surprising if we recall that minority voting rules, different from majority voting, do not result in long-range correlations. 
Hence, we can conclude that the quintuplet approximation that covers also the second-order neighborhood, is accurate enough to describe the dynamics of the CA on the macroscopic level. While it is of course understandable, that an approximation that considers more information is usually more accurate, we should also relate this conclusion to the computational effort. Here, it turns out that the 2nd-order approximation, although stochastic, i.e., needs to be averaged over a number of runs, performs very fast because of the closed form dynamics. Of course, the 1st-order approximation, the closed form of which is given in the Appendix, is computationally even simpler. But there is a trade-off with the accuracy of the prediction. Still, as long only majority voting is considered, the 1st-order approximation should be preferred, both for simplicity, accuracy and computational effort.

Our last remark is about the coexistence of the two opinions, which is the more interesting scenario compared to consensus, i.e., the existence of only one opinion. Here, we are not so much interested in the trivial case of random coexistence without any structure formation, which is characterized by $\langle x\rangle=0.5$ and $c_{1 \mid 1}=0.5$. We focus more on the case of correlated coexistence which has an interesting complexity because of the formation of local structures, nicely shown in Figure 2 (lower left). On the level of our approximations, this state is characterized by $\langle x\rangle=0.5$ and $c_{1 \mid 1} \rightarrow 1$. i.e., both opinions form at times large domains, indicated by the high pair correlation, but none of the two opinions entirely dominates the dynamics, as the expected value for its fraction is about 0.5 .

Such insights can be generalized to other cases of frequency dependent processes which e.g., play a role in population ecology (invasion or extinction of species). For most of these applications a two-dimensional CA is more appropriate as it was discussed in [8]. The one-dimensional CA investigated here, on the other hand, allows a mathematical approximation of the stochastic dynamics in terms of the 2nd-order neighborhood, which gives a much higher predictive power. This formalism can be also used for other frequency dependent processes in one-dimensional CA.

\section{Acknowledgments}

The authors acknowledge fruitful discussions with H. Mühlenbein on an early version of this paper.

\section{Author Contributions}

Both authors designed the research and wrote the paper. Laxmidhar Behera performed all numerical computations. Both authors have read and approved the final manuscript.

\section{Conflicts of Interest}

The authors declare no conflict of interest.

\section{Appendix}

In [8], we describe a method to calculate $\langle x\rangle, c_{1 \mid 1}$ and $\left\langle x_{1,1}\right\rangle$ for a two-dimensional non-linear VM. Here, we apply this approach to the one-dimensional CA, i.e., we consider a cell and its two nearest 
neighbors in pair approximation, using the transition rates of Equation (18). For simplicity, we use again the notation $x \equiv\langle x\rangle$. With Equation (30), we find for Equation (31)

$$
\frac{d x}{d t}=\varepsilon\left[\frac{1}{1-x}\left(1-2 x+x c_{1 \mid 1}\right)^{2}-x c_{1 \mid 1}^{2}\right]+\frac{(1-3 \alpha) x(2 x-1)\left(1-c_{1 \mid 1}\right)^{2}}{1-x}
$$

We note that $c_{1 \mid 1}=x$ if all neighboring states are uncorrelated, in this case Equation (A1) reduces to the mean-field Equation (25).

In order to calculate the time derivative of the doublet frequency $\left\langle x_{1,1}\right\rangle$ we have to consider how it is affected by changes of $\sigma$ in a specific opinion pattern $\left\{\sigma, \sigma_{1}, \sigma_{2}\right\}$, where the $\sigma_{j}$ refer to the given states of the $m=2$ neighbors of a cell. In a frequency dependent process it is assumed that the transition does not depend on the exact distribution of the $\sigma_{j}$, but only on the frequency of a particular state $\sigma$ in the neighborhood. Let $S_{\sigma, q}$ describe a neighborhood where the center cell in state $\sigma$ is surrounded by $q$ cells of the same state $\sigma$. For any given $q \leq m$, there are $\left(\begin{array}{c}m \\ q\end{array}\right)$ different opinion patterns with the same value of $q$. The global frequency to find a neighborhood $S_{\sigma, q}$ is denoted as $x_{\sigma, q}$ with the expectation value $\left\langle x_{\sigma, q}\right\rangle$. Regarding the possible transitions, we are only interested in changes of the doublet $(1,1)$, i.e., transitions $(1,1) \rightarrow(0,1)$ or $(0,1) \rightarrow(1,1)$. The transition rates shall be denoted as $w\left((0,1) \mid(1,1), S_{\sigma, q}\right)$ and $w\left((1,1) \mid(0,1), S_{\sigma, q}\right)$ respectively, which of course depend on the local neighborhood $S_{\sigma, q}$. The dynamics of the expected doublet frequency can then be described by the rate equation:

$$
\frac{d}{d t}\left\langle x_{1,1}\right\rangle(t)=\sum_{m=0}^{n-1}\left[w\left((1,1) \mid(0,1), S_{0, q}\right)\left\langle x_{0, q}\right\rangle-w\left((0,1) \mid(1,1), S_{1, q}\right)\left\langle x_{1, q}\right\rangle\right]
$$

In order to specify the transition rates of the doublets $w\left(\left(\sigma^{\prime}, 1\right) \mid(\sigma, 1), S_{\sigma, q}\right)$, with $\sigma^{\prime}=1-\sigma$ and $\sigma=\{(0,1)\}$, we note that there are only 6 distinct configurations of the neighborhood. Let us take the example $\underline{\sigma}^{0}=\{1,1,1\}$. A transition of the center cell $1 \rightarrow 0$ would lead to the extinction of 2 doublets $\sigma, \sigma_{j}=\{1,1\}$. On the other hand, the transition rate of the center cell is $\varepsilon$ as known from Equation (18). This would result in $w\left((0,1) \mid(1,1), S_{1,2}\right) \propto 2 \varepsilon$. For a lattice of size $N$ the number of doublets is $N$, so also the number of neighborhoods $\underline{\sigma}^{0}$ is $N$. Therefore, if we apply the transition rates of the single cells, Equation (18) to the transition of the doublets, their rates remain unchanged. Similarly, if we take the example $\underline{\sigma}^{0}=\{0,1,1\}$, a transition of the center cell $0 \rightarrow 1$ would occur at the rate $1-\alpha$ and would create 2 new doublets. We verify that $w\left((1,1) \mid(0,1), S_{0,0}\right)=2(1-\alpha)$. This way we can also determine the other possible transition rates:

$$
\begin{array}{ll}
\omega\left(01 \mid 11, S_{1,2}\right)=2 \varepsilon & \omega\left(01 \mid 11, S_{1,1}\right)=\alpha \\
\omega\left(01 \mid 11, S_{1,0}\right)=0 & \omega\left(11 \mid 01, S_{0,2}\right)=0 \\
\omega\left(11 \mid 01, S_{0,1}\right)=\alpha & \omega\left(11 \mid 01, S_{0,0}\right)=2(1-\alpha)
\end{array}
$$

Note that two of the transition rates are zero, because the respective doublets $(1,1)$ or $(0,1)$ do not exist in the assumed neighborhood.

Eventually, we express $\left\langle x_{\sigma, q}\right\rangle$ in Equation (A2) by

$$
\left\langle x_{\sigma, q}\right\rangle=\sum_{\underline{\sigma^{\prime}}}\left\langle x_{\sigma, \underline{\sigma^{\prime}}}\right\rangle
$$


and apply the pair approximation, Equation (27), to $\left\langle x_{\sigma, \underline{\sigma}^{\prime}}\right\rangle$, to obtain the dynamic equation for $\left\langle x_{1,1}\right\rangle$ :

$$
\begin{aligned}
\frac{d\left\langle x_{1,1}\right\rangle}{d t}= & +2 \alpha \frac{x}{(1-x)}\left(1-c_{1 \mid 1}\right)\left(1-2 x+x c_{1 \mid 1}\right)+2(1-\alpha) \frac{x^{2}}{(1-x)}\left(1-c_{1 \mid 1}\right)^{2} \\
& -2 \varepsilon x c_{1 \mid 1}{ }^{2}-2 \alpha x\left(1-c_{1 \mid 1}\right) c_{1 \mid 1}
\end{aligned}
$$

Finally, we obtain for the change of the correlation $c_{1 \mid 1}$ using Equations (32), (A1) and (A5):

$$
\begin{aligned}
\frac{d c_{1 \mid 1}}{d t}= & -\varepsilon\left[\frac{c_{1 \mid 1}}{x(1-x)}\left(1-2 x+x c_{1 \mid 1}\right)^{2}+c_{1 \mid 1}{ }^{3}-2 c_{1 \mid 1}{ }^{2}\right] \\
& +\frac{\left(1-c_{1 \mid 1}\right)^{2}\left(-2 \alpha-c_{1 \mid 1}+3 \alpha c_{1 \mid 1}-2(1-3 \alpha)\left(1-c_{1 \mid 1}\right) x\right)}{1-x}
\end{aligned}
$$

Thus Equations (A1) and (A6) are the final closed form for the dynamics of the one-dimensional CA. If we set $\alpha=0, \varepsilon=0$, then we get following simple macroscopic description for deterministic majority voting:

$$
\begin{aligned}
\frac{d x}{d t} & =\frac{x}{1-x}(2 x-1)\left(1-c_{1 \mid 1}\right)^{2} \\
\frac{d c_{1 \mid 1}}{d t} & =\frac{\left(1-c_{1 \mid 1}\right)^{2}}{1-x}\left[2 x-c_{1 \mid 1}(2 x-1)\right]
\end{aligned}
$$

\section{References}

1. Brown, D.T. A note on approximations to discrete probability distributions. Inf. Control 1959, 2, 386-392.

2. Mühlenbein, H.; Höns, R. Stochastic analysis of cellular automata with applications to the voter model. Adv. Complex Syst. 2002, 5, 301-337.

3. Pfante, O.; Bertschinger, N.; Olbrich, E.; Ay, N.; Jost, J. Comparison between different methods of level identification. Adv. Complex Syst. 2014, 17, 1450007.

4. Görnerup, O.; Jacobi, M.N. A method for inferring hierarchical dynamics in stochastic processes. Adv. Complex Syst. 2008, 11, doi:10.1142/S0219525908001507.

5. Banisch, S. From Microscopic Heterogeneity to Macroscopic Complexity in the Contrarian Voter Model. Adv. Complex Syst. 2004, 17, 1450025.

6. Agapie, A.; Höns, R.; Mühlenbein, H. Markov chain analysis for one-dimensional asynchronous cellular automata. Methodol. Comput. Appl. Probab. 2004, 6, 181-201.

7. Gleeson, J.P. Binary-state dynamics on complex networks: Pair approximation and beyond. Phys. Rev. X 2013, 3, 021004.

8. Schweitzer, F; Behera, L. Nonlinear voter models: The transition from invasion to coexistence. Eur. Phys. J. B 2009, 67, 301-318.

9. Krause, S.M.; Böttcher, P.; Bornholdt, S. Mean-field-like behavior of the generalized voter-model-class kinetic Ising model. Phys. Rev. E 2012, 85, 031126.

10. Stauffer, D. Better be third than second in a search for a majority opinion. Adv. Complex Syst. 2002, 5, 97-100.

11. Suchecki, K.; Eguíluz, V.M.; San Miguel, M. Conservation laws for the voter model in complex networks. Europhys. Lett. 2005, 69, 228-234. 
12. Suchecki, K.; Eguíluz, V.M.; San Miguel, M. Voter Model Dynamics in Complex Networks: Role of Dimensionality, Disorder, and Degree Distribution. Phys. Rev. E 2005, 72, 036132.

13. Behera, L.; Schweitzer, F. On Spatial Consensus Formation: Is the Sznajd Model Different from a Voter Model? Int. J. Mod. Phys. C 2003, 14, 1331-1354.

14. Przybyła, P.; Sznajd-Weron, K.; Tabiszewski, M. Exit probability in a one-dimensional nonlinear $q$-voter model. Phys. Rev. E 2011, 84, 031117.

15. Castellano, C.; Muñoz, M.A.; Pastor-Satorras, R. Nonlinear $q$-voter model. Phys. Rev. E 2009, 80, 041129.

16. Stark, H.-U.; Tessone, C.J.; Schweitzer, F. Slower is faster: Fostering consensus formation by heterogeneous inertia. Adv. Complex Syst. 2008, 11, 551-563.

17. Xiong, F.; Liu, Y.; Zhu, J. Competition of Dynamic Self-Confidence and Inhomogeneous Individual Influence in Voter Models. Entropy 2013, 15, 5292-5304.

18. Niu, H.-L.; Wang, J. Entropy and Recurrence Measures of a Financial Dynamic System by an Interacting Voter System. Entropy 2015, 17, 2590-2605.

19. Banisch, S.; Araújo, T.; Louçã, J. Opinion dynamics and communication networks. Adv. Complex Syst. 2010, 13, 95-111.

20. Keitt, T.H.; Lewis, M.A.; Holt, R.D. Allee Effects, Invasion Pinning, and Species' Borders. Am. Nat. 2001, 157, 203-216.

21. Castellano, C.; Fortunato, S.; Loreto, V. Statistical physics of social dynamics. Rev. Mod. Phys. 2009, 81, 591-646.

(c) 2015 by the authors; licensee MDPI, Basel, Switzerland. This article is an open access article distributed under the terms and conditions of the Creative Commons Attribution license (http://creativecommons.org/licenses/by/4.0/). 